krystallisiert mehrmals aus einem Gemisch von Chloroform, Aether und Alkohol um.

Das Stigmasterintetrabromid schmilzt bei $205^{\circ}$ unter Bräunung und Zersetzung. Es ist leicht löslich in Chloroform und Benzol, schwerer in Aether, sehr schwer in kaltem Eisessig und Alkohol, in der Hitze leichter. Zur Analyse wurde die Substanz bei $100^{\circ}$ getrocknet und folgendes Resultat erhalten :

0,1902 g Substanz gaben 0,1923 g AgBr.

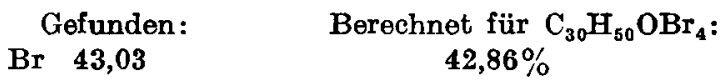

Mit diesem Tetrabromid wurden in gleicher Weise, wie vorher bei Sitostenon beschrieben, Oxydationsversuche angestellt und dabei ein Produkt mit einem Schmelzpunkt von $140^{\circ}$ erhalten. Dieser Stoff krystallisiert in sehr schön ausgebildeten Krystallen, die sich in Chloroform und Aether sehr leicht, in Benzol leicht, in Aceton schwerer, in kaltem Eisessig, in Aethyl- und Methylalkohol sogar sehr schwer lösen. Er zeigte keinen Ketoncharakter, konnte aber wegen Mangel an Ausgangsmaterial nur in sehr geringer Menge erhalten und daher vorläufig nicht weiter untersucht werden.

\title{
Das Benzoperoxyd.
}

(Eine Monographie mit eigenen experimentellen Versuchen.)

\author{
Von L. Van in o und F. Herzer.
}

(Eingegangen den 17. IX. 1915.)

Von den organischen Peroxyden, welche in letzterer Zeit wesentlich an Interesse gewonnen haben, ist das Benzoperoxyd, auch Benzoylsuperoxyd genannt, das bekannteste und als Repräsentant der organischen Peroxyde zu bezeichnen.

B rodi $e^{1}$ ) war der erste, der es darstellte. Er benutzte hierzu Chlorbenzoyl und Baryumsuperoxyd, indem er beide Komponenten mit Wasser mischte und das erhaltene Produkt mit wasserfreiem Aether auszog. Später beschäftigten sich mit der

1) Proc. Roy. Soc. XII., 655; Ann. 108, 80. 
Herstellung dieses Stoffes eine Reihe von Chemikern, wie L i p p mann, Sonnensohein, Erlenmeyer, von Pechmann, Vanino, Loewenhart, Gambarjan, Pribeshajew usw. Sonnenschein ${ }^{1}$ ) arbeitete mit Baryumsuperoxydhydrat, indem er das Benzoylchlorid in Wasser suspendierte und das genannte Hydrat unter Schütteln eintrug. Nach seinen Angaben erhielt er auf diese. Weise 65\% Ausbeute. Erlenm e y e $r^{2}$ ) ging vom Benzaldehyd aus, indem er dasselbe mit Essigsäureanhydrid und Sand mischte, eine Methode, die, wie die eben erwähnten, nur mehr historisches Interesse hat. Im Jahre 1894 veröffentlichten $v$ on $P$ e $\mathrm{chmann}$ und $\mathrm{Vanino}$ eine andere Methode zur Darstellung mittels Benzoylchlorid, Natronlauge und Wasserstoffsuperoxyd, deren sich auch $v$ on $B$ a e yer und V illig er bei ihren interessanten Arbeiten bedienten, und welche meist als Grundlage für die späteren Darstellungsweisen diente. Die Reaktion verläuft bei dieser Reaktion nach folgender Gleichung:

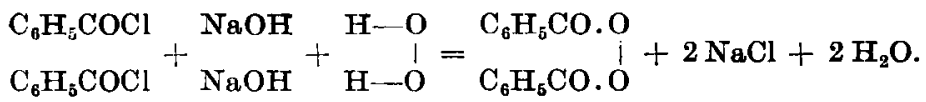

Später benutzten $\mathrm{V}$ a $\mathrm{n}$ in o und $\mathrm{E}$. $\mathrm{Th}$ i e $\mathrm{e}^{3}$ ) das Natriumsuperoxydhydrat, indem sie z. B. $20 \mathrm{~g}$ Natriumsuperoxyd in 150 bis $200 \mathrm{ccm}$ auf $0^{\circ}$ abgekühltem Wasser lösten und in die Lösung $50 \mathrm{~g}$ Benzoylchlorid unter fortwährendem Umrühren eintrugen. Auch $\mathrm{Nenki}$ und $\mathrm{Zaleski}$ ) wandten bei der Darstellung des Benzoperoxydes das Natriumsuperoxyd an. In ähnlicher Weise experimentierte A. L. Loewenhart. Stephan Gam b a r j a $n^{5}$ ) gewinnt es, indem er $100 \mathrm{~g}$ Benzoylchlorid mit dem doppelten Volumen Aceton versetzte und die Mischung unter Turbinieren und Kühlen mit Eis zu einer Auflösung von $40 \mathrm{~g}$ Natriumsuperoxyd in $\mathbf{4 0 0} \mathrm{g}$ Eiswasser zutropfen ließ. N. P r i b e sh a j e w6) endlich, der sich ebenfalls mit der Darstellungsweise mittels Natriumsuperoxyd beschäftigte, bemerkt, daB die Ausbeute bis zu $100^{\circ}$ von einem rationellen Rühren abhängig ist, wobei ein Zusammenbacken des gebildeten Produktes zu vermeiden ist.

Unsere Versuche waren nun zuerst darauf gerichtet, die verschiedenen Arbeitsmethoden auf ihre Ausbeuten zu prüfen, da die

1) Monatsh. 7 (1886), 522; Anm.

2) Ber. 27 (1894), 1959.

3) Ber. 29 (1896), 1724.

4) Ztschr. physiol. Chem. 27 (1899), 487.

5) Ber.' 42 (1909), 4003.

6) Journ. russ. physiol. Ges. 42 (1910), 138 i. 
Angaben hierüber ziemlich stark auseinandergehen. Wie schon erwähnt, gab S o n nens che in $65 \%$ Ausbeute an, von Pechmann und Vanino erhielten $70 \%$, von Baeyer und Villiger, welche das Produkt nicht aus Alkohol umkrystallisierten, sondern in Chloroform lösten und mit Methylalkohol fällten, bezeichnen die Ausbeute so gut wie quantitativ. S t $\Theta \mathrm{p} \mathrm{h}$ a $\mathrm{n}$ Ga m barjan erhielt 71\%, und. nach Pribeshajew's Angaben war die Ausbeute nach seinem Verfahren ebenfalls quantitativ.

Versuche in bezug auf die Ausbeuten ergaben nur geringe Abweichungen in den angegebenen Zahlen; nur bei Anwendung des technischen Benzoylchlorids zeigten sich ziemliche Differenzen in den Methoden selbst. So ergab z. B. die $\nabla$. P e chmann$\mathrm{V}$ a $\mathrm{n}$ i n o sche Methode beim reinen Benzoylchlorid nahezu 85\%, beim technischen dagegen $\mathbf{7 4 , 4} \%$.

Es wurden ferner auch Versuche mit Natriumperborat gemacht. Dasselbe reagiert bekanntlich mit Wasser unter Bildung von Wasserstoffdioxyd. Der Vorgang kann durch folgende einfache Gleichung dargestellt werden:

$$
\mathrm{NaBO}_{3}+\mathrm{H}_{2} \mathrm{O}=\mathrm{NaBO}_{2}+\mathrm{H}_{2} \mathrm{O}_{2} \text {. }
$$

Es ergibt sich also, da das genannte Perborat mit vier Wasser krystallisiert, das Verhältnis $154: 281$. Wie vorauszusehen war, zelang es auf diese Weise Benzoperoxyd darzustellen, jedoch war die Ausbeute nicht zufriedenstellend. Sie betrug nur $30^{\circ}$ der Theorie.

\section{Eigensehaften des Benzoperoxydes.}

Das Benzoperoxyd ist ein schön krystallisierender Stoff, der in reinem Zustande einen etwas an Chlorkalk erinnernden Geruch besitzt. In Wasser ist es so gut wie unlöslich. Spuren scheinen sich in demselben zu lösen, da eine alkalische verdünnte Goldchloridlösung von demselben zu kolloidem Gold wird. Aus Alkohol, Essigester, Chloroform und Schwefelkohlenstoff ist es unkrystallisierbar, da es von diesen Lösungsmitteln nur in der Wärme aufgenommen wird. Aceton, Aether, Eisessig, Benzol, Toluol, Nitrobenzol und Pyridin lösen den Körper auch in der Kälte in ziemlichen Mengen auf, während er in Methylalkohol, Benzin und Gasolin nur schwer löslich ist; auch von Petroleum wird er in der Wärme leicht aufgenommen und krystallisiert in der Kälte wieder aus. Zur Reindarstellung eignen sich insbesondere, wie oben angegeben, Aethylalkohol, Essigester, Chloroform und Schwefelkohlenstoff; in sehr reiner Form erhält man die Substanz auch, 
wenn man dieselbe in Chloroform ${ }^{1}$ ) löst und mit Methylalkohol daraus ausfällt. Die Krystalle, die aus Alkohol und Essigester ausfallen, sind prismatisch, während cie in Chloroform und Schwefelkohlenstoff eine mehr gedrungene Gestalt annehmen und deutlich ihre Zugehörigkeit zum rhombischen System erkennen lassen. Ueber ihre physikalischen Eigenschaften machte seinerzeit B.r od i $e^{2}$ ) nähere Mitteilungen.

Der Schmelzpunkt des Benzoylsuperoxydes wird verschieden angegeben, was wohl davon herkommen mag, daß es bei dieser Substanz von wesentlichem Einflusse ist, ob man langsam oder rasch erhitzt. B r od i $e^{2}$ gibt $103,5^{0}$ an, ebenso $P e c h m$ a $n$ und $V$ a $n$ in ${ }^{3}$ ); nach $B$ a $\theta$ yer und Villige $\mathrm{r}^{4}$ ) schmilzt das nach ihrer Methode umkristallisierte Produkt bei 106-1080. Bei erneuten Versuchen, die wir in bezug auf den Schmelzpunkt unternahmen, zeigte ein zweimal aus Alkohol umkrystallisiertes und mit Aether gewaschenes Produkt einen Schmelzpunkt von $104-105^{\circ}$.

Beim Erhitzen in der Flamme verpufft das Benzoperoxyd, eine Eigenschaft, die es mit allen bis jetzt dargestellten Vertretern dieser Körperklasse teilt, und die bei einigen, wie beim Acetyl-, Succinyl-, Phthalylperoxyd und anderen, schon bei geringem Druck zutage tritt. Dieses Verpuffen kann man bedeutend lebhafter gestalten, wenn man gewisse sauerstoffabgebende Mittel zusetzt. So zeigt ein Gemisch von Benzoperoxyd mit feingepulvertem Kaliumpermanganat beim Erhitzen im Reagenzrohr eine lebhafte Flammenerscheinung, ebenso wenn man statt des Permanganats Natriumsuperoxyd anwendet; dagegen ist bei Anwendung von Kaliumnitrat oder Kaliumchlorat die Verpuffung nicht heftiger als ohne Zusatz; auch wenn man durch -Zugabe von Braunstein die Zersetzung des Kaliumchlorates zu erleichtern sucht, erhält man keine kräftigere Wirkung. Ebenso erwiesen sich Baryumsuperoxyd, Baryumchlorat, Kaliumbromat und Kaliumjodat ohne Einfluß auf die Heftigkeit der Zersetzung des Superoxyds.

Eine sehr kräftige Reaktion tritt ein, wenn man das Benzoperoxyd mit Cyankali ${ }^{5}$ mischt; beim Erhitzen explodiert das Gemenge mit starkem Knall.

1) Ber. 33 (1900), 1575.

2) Ann. III., Suppl. 207.

3) Ber. 27 (1894), 1510.

4) Ber. 33 (1900), 1575 .

s) Ber. 30 (1897), 2003. 
Die explosionsartige Zersetzung des Benzoperoxyds durch Wärmezufuhr kann man mäßigen, wenn man dasselbe in feinverteiltem Zustande mit Sand ${ }^{1}$ ) mischt: unter diesen Umständen geht die Zersetzung des Körpers sehr ruhig von statten, und es wird Kohlensäure dabei entwickelt.

Während die anorganischen Superoxyde leicht mit Salzsäure unter Chlorentwickelung reagieren, ist die große Beständigkeit des Benzoperoxyds und anderer organischer Superoxyde gegenüber diesem Reagens sehr bemerkenswert. Weder beim Kochen mit der konzentrierten Säure bei gewöhnlichem Druck, noch bei erhöhtem Druck und gesteigerter Temperatur konnten wir eine Einwirkung der Säure auf das Superoxyd nachweisen. Um die Wirkung der Salzsäure bei hohem Druck und hoher Temperatur festzustellen, schlossen wir $0,4 \mathrm{~g}$ der Substanz mit $5 \mathrm{ccm}$ der konzentrierten Säure im Bombenrohr ein und erhitzten zuerst zwei Stunden auf $120^{\circ}$, dann drei Stunden auf $150^{\circ}$ und endlich fünf Stunden auf $200^{\circ}$ : beim Oeffnen der Röhre konnten wir in keinem Falle den Geruch von Chlor wahrnehmen; das Benzoperoxyd war nur an einigen Stellen infolge geringer Verkohlung bräunlich gefärbt. Ebenso beständig erwies sich dasselbe, wie vorauszusehen war, gegen Fluorwasserstoffsäure; auch hier konnte ich eine Oxydationswirkung nicht feststellen. Dagegen reagiert das Superoxyd mit Bromwasserstoffsäure und besonders leicht mit Jodwasserstoffsäure. Wenn man zu einer wässerigen Lösung ron Bromkali oder Jodkali feinverteiltes Benzoperoxyd zugibt und einige Zeit stehen läßt, so bemerkt man zunächst bei der Jodkalilösung eine schwache Gelbfärbung infolge Jodausscheidung; die Bromkalilösung dagegen bleibt in der Kälte farblos und erst in der Wärme tritt schwache Bromabscheidung ein. Beide Reaktionen gehen auf diese Weise nur äußerst langsam und unvollständig von statten; man kann sie aber beschleunigen, insbesondere beim Jodkali, wenn man Alkohol oder Aceton zugibt, die auf das Benzoperoxyd lösend einwirken. Auf diese Weise gehen beide Reaktionen bei mäßigem Erwärmen bedeutend rascher von statten und besonders die Jodabscheidung wird nach kurzer Zeit eine quantitative, so daß sie als Grundlage zu einer maßanalytischen Bestimmung des Benzoperoxyds dienen kann. Darüber wird in einem späteren Abschnitt $\mathrm{zu}$ berichten sein.

Während also die Halogenwasserstoffsäuren, sofern sie überhaupt auf den Körper einwirken, durchaus keine stürmischen 
Reaktionen zeigen, gestaltet sich das Verhalten der konzentrierten Schwefelsäure gegenüber demselben bedeutend lebhafter. Wirft man trockenes Benzoperoxyd auf die konzentrierte Säure ${ }^{1}$ ), so tritt unter starkem Erwärmen ein lebhaftes Verpuffen ein und die Flüssigkeit nimmt unter Kohleabscheidung eine grtine Färbung an. Verdünnt man die konzentrierte Säure mit demselben Volumen Wasser, so löst sich das Peroxyd ohne weiteres darin auf.

Verdünnte Salpetersäure zeigt auch 'bei mäßigem Erwärmen keine Einwirkung, während bei höherer Temperatur die Substanz sich verflüssigt und eine Explosion eintritt. Dagegen wird sie leicht von der konzentrierten rauchenden Säure aufgenommen unter Bildung von m-Nitrobenzoylperoxyd. Die Nitrierungsprodukte des Benzoperoxyds sollen weiter unten besprochen werden.

Schon aus dem indifferenten Verhalten der Substanz gegenüber der konzentrierten Salzsäure läßt sich ersehen, daß erstere weit beständiger ist, als man im Hinblick auf ihre Superoxydnatur erwarten sollte. Auch aus dem Verhalten gegen andere Reagentien tritt deutlich zutage, daß sie verhältnismäßig schwer ihren verfügbaren Sauerstoff abgibt; so tritt die Entfärbung von Permanganat, wenn man eine verdünnte Lösung dieses Salzes mit Benzoperoxyd kocht, nur sehr langsam ein; leichter geht die Reaktion von statten, wenn man das Peroxyd in mäBig konzentrierter Schwefelsäure löst und nach Zugabe einiger Tropfen Permanganatlösung schwach erwärmt; vielleicht beruht die Entfärbung in diesem Falle auf Anwesenheit von freiem Wasserstoffsuperoxyd, das sich beim Lösen des Benzoperoxyds in der Säure gebildet hat; daß in der Tat ein solcher Vorgang stattfindet, kann man aus der Tatsache schließen, daß eine Lösung der Substanz in mäßig konzentrierter Schwefelsäure mit Titanschwefelsäure die bekannte rotgelbe Färbung gibt, die als Reagens auf freies Wasserstoffsuperoxyd dient. Allerdings konnten wir die andere Reaktion auf $\mathrm{H}_{2} \mathrm{O}_{2}$, die auf der Bildung der Ueberchromsäure beruht, in diesem Falle nicht bekommen.

Des weiteren suchten wir salpetrige Säure auf das Benzoperoxyd einwirken zu lassen. Wir gingen in der Weise vor, daß wir eine Suspension von feinverteiltem Peroxyd in angesäuerter Natriumnitritlösung mehrere Stunden unter guter Eiskühlung stehen ließen; auch in diesem Falle zeigte die Substanz eine große Beständigkeit, denn es konnte keinerlei Einwirkung festgestellt werden.

1) Ber. 30 (1897), 2004. 


\section{Verhalten gegen Reduktionsmittel.}

Reduktionsmittel, wie schweflige Säure und unterphosphorige Säure, ließen auch nach längerer Behandlung in der Kälte wie in der Wärme den Körper unverändert. Geringe Reduktionswirkungen zeigen sich bei Anwendung von Natriumamalgam, ferner wenn man mit Salzsäure und Zinkstaub kocht: in beiden Fällen kann man eine teilweise Reduktion zu Benzoesäure nachweisen; vertauscht man in letzterem Falle die Salzsäure gegen Eisessig, in welchem das Benzoperoxyd leicht löslich ist, so geht die Reduktion bedeutend leichter von statten. Sehr leicht läßt sich dagegen dem Körper der labile Sauerstoff entziehen durch Behandeln mit Zinnchlorür: wenn man zu einer alkoholischen Lösung der Substanz etwas Zinnchlorürlösung gibt und erwärmt, so tritt glatte Reduktion ein. Eine andere Methode das Benzoperoxyd zu Benzoesäure zu reduzieren, haben Willstätter und Hauenstein angegeben; sie ließen nämlich Wasserstoff unter dem katalytischen Einfluß von Platin auf die Substanz einwirken, und es gelang ihnen auf diese Weise dasselbe quantitativ zu Benzoesäure zu reduzieren.

Auch an einigen organischen Reduktionsmitteln suchten wir die Reaktionsfähigkeit des Körpers festzustellen. Läßt man auf denselben Formaldehyd ${ }^{1}$ ) in alkalischer Lösung einwirken, so kann man die Entwickelung von Kohlensäure nachweisen; wenn man statt des Formaldehyds Chloralhydrat anwendet, so läßt sich auch hier eine Oxydationswirkung feststellen. Experimentiert man zu diesem Zwecke mit $4 \mathrm{~g}$ Chloralhydrat in wässeriger Lösung und 20,5 g Benzoperoxyd drei Stunden am Rückflußkühler, so sammelte sich am Boden des Kölbchens allmählich ein rotgelbes Oel an, das beim Erkalten fest wurde und sich nach der Trennung von der Flüssigkeit als Benzoesäure, die durch teilweise verharztes Benzoperoxyd gefärbt war, erwies. Außerdem ließ sich der Geruch von Chloroform und die Entwickelung von Kohlensäure nachweisen. Man kann sich den Verlauf der Reaktion so vorstellen, da $B$ das Chloralhydrat zu Trichloressigsäure oxydiert wurde und diese dann weiterhin durch das Kochen in wässeriger Lösung in Chloroform und Kohlensäure zerfällt. Eine genauere Untersuchung der Reaktionsprodukte bei diesem, wie bei anderen Versuchen wurde nicht ausgeführt, da es uns nur darauf ankam, festzustellen, ob. eine Oxydationswirkung eintrat.

Bei mäBigem Erwärmen von Glycerin mit der Substanz. kann man deutlich Aldehydgeruch wahrnehmen, bei stärkerem

1) Ber. 30 (1897), 2003. 
E'rhitzen über freier Flamme tritt plötzlich unter starkem Knall und Rauchentwickelung eine heftige Reaktion ein.

Eine weitere Reaktion, die ebenfalls auf der Oxydationsfähigkeit des Benzoperoxyds beruht, haben seinerzeit V a n in o und $\mathrm{Thi} \in \mathrm{le}^{\mathrm{l}}$ ) angegeben; sie versetzten nämlich ätherische Phenylhydrazinlösung mit Benzoperoxyd; unter Erwärmung und Stickstoffentwickelung trat bald die Lösung desselben ein, und nach dem Verdunsten des Aethers krystallisierten zwei Körper aus, von denen der eine sich als Benzoesäure erwies, während der andere durch die Elementaranalyse als Monobenzoylphenylhydrazin identifiziert wurde. Auoh Hydroxylamin wird durch den Körper zu Stickstoff oxydiert; ferner erhält man eine Stickstoffentwickelung, wenn man alkoholisches Ammoniak einwirken läßt; als Eindprodukt erhält man hier benzoesaures Ammoniak; die Reaktion geht jedenfalls in der Weise vor sich, daß zunächst das Ammoniak zu Stickstoff oxydiert wird; das dabei entstehende Wasser verseift das überschüssige Benzoperoxyd zu Benzoesäure und diese vereinigt sich mit dem noch nicht oxydierten Ammoniak zu dem Salz.

Des weiteren ließen wir einige organische Süuren auf das Benzoperoxyd einwirken; dabei zeigte sich wieder die große Beständigkeit desselben, als wir die Substanz mit Ameisensäure behandelten. Obwohl doch diese Säure bekanntlich eine ziemlich große Reduktionskraft besitzt, vermochte sie doch nicht die Substanz zu verändern; vielmehr wird dieselbe in großen Mengen von der Säure gelöst und auch nach längerem Kochen in der Kältte unverändert wieder abgeschieden. Auch Oxalsäure und Zitronensäure zeigten nach dem Kochen in wässeriger oder alkoholischer Lösung mit der Substanz keine Einwirkung auf dieselbe.

So beständig sich auch das Benzoperoxyd in vielen Fällen gegen Oxydations- und Reduktionsmittel zeigt, so ist es andererseits doch sehr leicht, ihm den labilen Sauerstoff zu entziehen, nämlich wenn man es mit Kali- oder Natronlauge kocht; in diesem Falle zerfällt es glatt zu Benzoesäure, bzw. zu benzoesaurem Alkali und Sauerstoff; noch leichter vollzieht sich diese Reaktion, venu man statt der wässerigen Lauge alkoholische Lauge anwendet: man braucht dann nur gelinde zu erwärmen, um die Zersetzung des Körpers unter kräftiger Sauerstoffentwickelung zu bewirken. Der Vorgang läßt sich durch folgende Formel veranschaulichen:

$$
\begin{aligned}
& \mathrm{C}_{6} \mathrm{H}_{5} \mathrm{CO}-\mathrm{O} \\
& \mathrm{C}_{3} \mathrm{H}_{5} \mathrm{CO} \quad \mathrm{O}+4 \mathrm{KOH}=4 \mathrm{C}_{6} \mathrm{H}_{5} \mathrm{COOK}+2 \mathrm{H}_{2} \mathrm{O}+\mathrm{O}_{2}
\end{aligned}
$$

1) Ber. 29 (1896), 1725. 
Benzoperoxyd als Sehwefel verdrängendes Mittel.

In neuerer Zeit hat der eine von uns im Verein mit A. Sch innerl) gezeigt, daß mittels des Benzoperoxyds geschwefelten Ketonen und ähnlichen Thiokarbonylverbindungen der Schwefel auch entzogen werden kann. 'Zu diesen Versuchen wählten sie zunächst den Thioharnstoff als Untersuchungsobjekt; es zeigte sich dabei, daß bei Anwendung höherer Temperatur die Entschwefelung des Thioharnstoffs nahezu quantitativ ist. Um zu untersuchen, ob sich diese Eigenschaft des Benzoperoxyds auch auf Substitutionsprodukte des Thioharnstoffs erstrecke, unterwarfen wir o- und p-Tolylsulfoharnstoff der Behandlung mit demselben, indem wir die alkoholische Lösung des Superoxyds mit der entsprechenden Menge der geschwefelten Verbindung zusammengaben und mehrere Tage stehen ließen. Auch hier konnte die Schwefelabscheidung sofort bei der Mischung der beiden Komponenten in beiden Fällen beobachtet werden, und nach Ablauf von 14 Tagen hatten sich am Boden der Gefäße schöne rhombische Schwefelkrystalle ausgeschieden. Auch Diphenylsulfoharnstoff läßt sich auf diese Weise entschwefeln.

Wir versuchten nun auch auf eine anorganische Schwefelverbindung diese Eigenschaft des Peroxydes auszudehnen und wählten hierzu das Natriumthiosulfat. Es zeigte sich dabei, daB die Entschwefelung in wässeriger Lösung nicht gelingt, schmilat man dagegen das Thiosulfat in seinem Krystallwasser und gibt Benzoperoxyd zu, so tritt nach kurzer Zeit kräftige Schwefelabscheidung ein. Da diese Reaktion sich jedoch erst bei höherer Temperatur einstellt, so kommt es leicht vor, daß das noch überschüssige Peroxyd unter Verpuffen sich zersetzt; es ist daher sehr schwierig, diese Reaktion nach ihrer quantitativen Seite zu untersuchen, und sie hat daher nur experimentelles Interesse.

\section{Weitere, in der Literatur sich findende Reaktionen des Benzoperoxyds.}

Von hohem Interesse sind noch einige andere in der Literatur bereits bekanntgegebene Reaktionen. So haben Ba eyer und Villige $\mathrm{r}^{2}$ ), die sich ebenfalls mit ihm beschäftigten, die interessante Beobachtung gemacht, daß bei der Behandlung der ätherischen Lösung des Peroxyds mit Natriumalkoholat das

1) Ber. 47 (1914), 699.

2) Ber. 33 (1900), 1575. 
Natriumsalz der Benzopersäure ausfällt, während Benzoesäureäthylester in Lösung bleibt:

$$
\begin{aligned}
& \mathrm{C}_{6} \mathrm{H}_{5} \mathrm{CO}-\mathrm{O} \\
& \mathrm{C}_{6} \mathrm{H}_{5} \mathrm{CO}-\mathrm{O}
\end{aligned}+\mathrm{NaOC}_{2} \mathrm{H}_{5}=\mathrm{C}_{6} \mathrm{H}_{5} \mathrm{CO}-\mathrm{O}-\mathrm{ONa}+\mathrm{C}_{6} \mathrm{H}_{5} \mathrm{COOC}_{2} \mathrm{H}_{5} \text {. }
$$

E. L i p p m a $\mathbf{n}^{1}$ ) benutzte das Benzoperoxyd bei der Abspaltung von Wasserstoff bei den aromatischen Kohlenwasserstoffen. Er erhitzte Benzol mit Benzoperoxyd im Bombenrohr auf 100 bis $140^{\circ}$ und exhielt dabei Kohlensäure, Benzoesäure und Diphenyl:

$$
\mathrm{C}_{6} \mathrm{H}_{5} \mathrm{CO}-\mathrm{O} \mathrm{H}_{6} \mathrm{CO} \stackrel{\mathrm{O}}{\mathrm{O}}+\mathrm{C}_{6} \mathrm{H}_{6}=\mathrm{C}_{6} \mathrm{H}_{5} \mathrm{COOH}+\mathrm{CO}_{2}+\underset{\mathrm{C}_{6} \mathrm{H}_{5}}{\mathrm{C}_{6} \mathrm{H}_{5}}
$$

$\mathrm{S}$ t. Gam $\mathrm{b}$ a $\mathbf{r} \mathrm{j} \mathbf{n}^{2}$ ) löste Benzoperoxyd in Chloroform und ließ darauf Diphenylamin einwirken; er erhielt auf diese Weise N-Benzoyl-o-oxydiphenylamin.

\section{Ueber die Nitrierung des Benzoperoxyds.}

Wie bereits erwähnt, erhält man durch Behandlung von Benzoperoxyd mit rauchender Salpetersäure das m-Nitrobenzoylsuperoxyd. Diese Verbindung wurde zuerst von $\mathrm{Brodie}{ }^{3}$ ) beschrieben. Er stellte es dar, indem er Benzoperoxyd mit der genügenden Menge von Salpetersäure vom spezifischen Gewicht 1,505 übergoß und 24 Stunden stehen ließ. Eine Methode, die weniger Zeit in Anspruch nimmt, hat $V$ an in $0^{4}$ ) angegeben. Er gibt in kalte, konzentrierte Salpetersäure Benzoperoxyd in genügender Menge und erwärmt etwa dreiviertel Stunden auf dem Wasserbad. Die Reaktion verläuft gefahrlos und unter starker Gasentwickelung. Nach dem Erkalten fällt das Nitroprodukt durch Zugabe von viel Wasser aus.

Beide Methoden unterzogen wir nochmals einer Untersuchung, da in der Literatur bezüglich der Ausbeuten usw. keinerlei Angaben vorhanden sind. Bei der Arbeitsweise von B r odie erhielten wir aus $10 \mathrm{~g}$ Benzoperoxyd $5,7 \mathrm{~g}$ reines Nitroprodukt, d. h. $42 \%$ Ausbeute. Darauf wandten wir die $\mathrm{r}$ a $n$ in o'sche Methode an, konnten jedoch mit der reinen konzentrierten Salpetersäure keine befriedigenden Resultate erzielen: der mit Wasser entstehende Niederschlag enthielt zum größten

1) Ber. 19 (1886), 744.

2) Ber. 42 (1909), 4008.

3) Ann. III., Suppl. 209

4) Ber. 30 (1897), 2004. 
Teil Benzoesäure bzw. Nitrobenzoesäure, während Nitrobenzoperoxyd nur in geringer Menge sich vorfand. Um die Ausbeute zu verbessern, versuchten wir die rauchende Säure und erhielten auf diese Weise gute Resultate. Der Arbeitsgang gestaltet sich am besten folgendermaßen: $10 \mathrm{~g}$ Benzoperoxyd gibt man in kleinen Mengen zu $150 \mathrm{ccm}$ der rauchenden Säure, wobei man jedesmal gut umschüttelt und wartet bis sich alles gelöst hat; das Peroxyd löst sich leicht und unter starker Entwickelung von Stickoxyden auf. Wenn man in kleinen Mengen zusetzt, so ist die Erwärmung nicht zu stark, so daß ein Abkühlen nicht notwendig ist. Wenn alle Substanz zugegeben ist, läßt man erkalten und gießt dann das Reaktionsgemisch in dünnem Strahl unter stetem Umrühren in etwa 1 Liter mit Eisstücken versetztes Wasser, wobei ein gelblicher Niederschlag entsteht, der sich meistens zu harten Brocken zusammenballt. Man läßt einige Zeit stehen, filtriert $a b$ und kocht den Rückstand, nachdem man ihn mit einem Pistill zerdrückt hat, mit heißem Wasser mehrmals aus, um die nebenbei entstehende Benzoesäure und Nitrobenzoesäure zu entfernen. Der Rückstand wird auf Ton im Exsikkator getrocknet und aus Essigester umkrystallisiert. Man erhält so das m-Nitrobenzoperoxyd in feinen Nädelchen, die bei $139-140^{\circ}$ unter Zersetzung schmelzen. Die Ausbeute beträgt bei dieser Methode etwa 50\% der Theorie.

Wenn man statt der rauchenden Salpetersäure allein oin Gemisch derselben mit konzentrierter Schwefelsäure anwendet, eine Arbeitsweise, die seinerzeit von $\mathrm{V}$ a $\mathrm{n}$ i $\mathrm{n}$ o und $\mathrm{U} \mathrm{h} \mathrm{l}$ f e $1 \mathrm{de} \mathbf{r}^{\mathbf{1}}$ ) angegeben wurde, so kann man die Ausbeute bis zu $65 \%$ steigern. Hier muß man besonders auf gute Kühlung achten, vor allem zu Anfang, da sonst die Reaktion leicht einen explosionsartigen Verlauf nimmt. Die Arbeitsweise ist sonst die gleiche wie oben.

Das p-Nitrobenzoperoxyd erhält man bekanntlich durch Einwirkung von Wasserstoffsuperoxyd auf p-Nitrobenzoylchlorid bei Gegenwart von Pyridin²). Hier erhielten wir 52\% Ausbeute. Die Darstellung geht recht glatt von statten.

\section{Analytisches iiber das Benzoperoxyd.}

Benzoperoxyd reduziert Goldlösungen und scheidet aus Jodl:alium Jod aus. Diese Eigenschaften hat es mit Wasserstoffsuperoxyd und anderen Peroxyden gemein. Eine spezifische

1) Ber. 33 (1900), 1046.

2) Ber. 33 (1900), 1046. 
Reaktion des Benzoperoxyds tritt nach L. G o l odetz ein, wenn man dasselbe mit konzentrierter Schwefelsäure und Formaldehyd behandelt: es tritt Rotfärbung auf. Diese Reaktion kann zum Nachweis von Formaldehyd benutzt werden. Gibt man nämlich einige Körnchen Benzoperoxyd in 10-12 Tropfen konzentrierte Schwefelsäure, so zersetzt es sich unter Verpuffen und Bildung von weißen Dämpfen, und es tritt ein Geruch von Benzophenon auf. Die so erhaltene Mischung färbt sich auf Zusatz von wenig Formaldehydlösung sofort beständig blutrot. Wasser bringt die Färbung zum Verschwinden. Die beschriebene Farbenerscheinung ist sehr empfindlich, so daß sie sich noch mit einer $0,04 \%$ igen Formaldehydlösung erkennen läßt.

Ferner läßt sich das Benzoperoxyd nach einer Mitteilung aus E. M e r c k's Jahresberichten (1909) auch zum Nachweis von Cholesterin verwenden. Das Verfahren ist folgendes: Eine Lösung von einigen Milligrammen Cholesterin in 2-3 ccm Eisessig wird nit einigen Körnchen. Benzoperoxyd versetzt und einmal aufgekocht. Sobald sich das Gemisch abgekühlt hat, gibt man einige Tropfen konzentrierter Schwefelsäure zu, die sich am Boden des Reagenzglases ansammeln und eine blauviolette bis blaugrüne Farbe annehmen.

\section{Ueber eine neue quantitative Bestimmung des Benzoperoxyds.}

Ueber eine quantitative Bestimmung des Benzoperoxyds finden sich in der Literatur noch keine Angaben. Wir suchten daher eine Methode ausfindig zu machen, die es erlaubt, dasselbe schnell und sicher quantitativ zu bestimmen. Am nächsten kamen wir diesem Ziele bei der Anwendung der jodometrischen Methode.

Uebergießt man feinverteiltes Benzoperoxyd mit Jodkalilösung und läßt stehen, so tritt nach einiger Zeit schwache Gelbfärbung ein. Diese Reaktion verläuft jedoch, wie schon oben erwähnt wurde, unter diesen Umständen so langsam, daß selbst nach tagelangem Stehen die Jodausscheidung nur eine geringe, geschweige denn eine quantitative ist; auch durch Erwärmen wird die Reaktion nur wenig beschleunigt. Sobald man aber ein Lösungsmittel zugibt, das mit Wasser mischbar ist und lösend auf das Benzoperoxyd einwirkt, also z. B. Alkohol, so erfolgt nach kurzer Zeit starke Jodausscheidung. Im Hinblick auf dieses Verhalten geht man bei dieser Bestimmungsweise folgendermaßen vor: Man wägt $0,2-0,3 \mathrm{~g}$ trockenes, vollkommen reines Benzoporoxyd mittels eines Wägegläschens in einen kleinen Erlenmeyer ab, gibt 10-15 ccm Alkohol hinzu und erwärmt kurze Zeit auf dem Wasser- 
bad, bis sich alles gelöst hat. Dann setzt man eine überschüssige Menge Jodkalilösung zu und säuert schwach an. Die Jodkalilösung soll nicht zu verdünnt sein, da sonst leicht ein Teil des Benzoperoxyds aus der alkoholischen Lösung ausfält; wir verwendeten eine $1 / 2-N$.-Lösung. Nun erhitzt man das Kölbchen noch vier Minuten auf dem Wasserbade und läßt dann erkalten; nach einer halben Stunde kann man dann mit einer frisch eingestellten Thiosulfatlösung titrieren. Daß diese Methode recht genaue Resultate ergibt, läßt sich aus folgenden Angaben von zehn Titrationen ersehen:

$$
\text { Analyse No. }
$$

I.

$$
\text { II. }
$$

III.

IV.

V.

Abgewogen Gramm . . 0,1611

Gefunden Gramm . . . 0,1608

Prozent

$\begin{array}{cccc}0,1735 & 0,1851 & 0,1817 & 0,1906 \\ 0,1734 & 0,1850 & 0,1818 & 0,1901 \\ 99,94 & 99,95 & 100,1 & 99,74\end{array}$

Analyse No. 99,81

99,94 $\mathbf{9 9 , 9 5}$ 100,1 99,74

Abgewogen Gramm . . . 0,1771

Gefunden Gramm . . 0 0,1771

VII.

VIIT.

IX.

$\mathrm{X}$. Prozent 100

$0,1777 \quad 0,2032$

0,1961

0,1716

0,1776

0,1961

0,1713 $\mathbf{9 9 , 9 4} \quad 99,85$

100 99,82

Im Mittel wurden also 99,94 des abgewogenen Benzoperoxyds durch die Titrationen gefunden.

Auch mit Kaliumpermanganat versuchten wir das Benzoperoxyd zu titrieren, jedoch konnten wir bei diesen Versuchen keine befriedigenden Resultate erhalten.

Endlich zogen wir noch die Methode der Benzoylbestimmung von A. W. van der $\mathrm{H}$ a a r $\mathbf{r}^{\mathbf{1}}$ ) zum Vergleich heran. Sie beruht auf der Verseifung der Benzoylderivate mit alkoholischer Kalilauge und Wägung der entstandenen Benzoesäure. Diese Verseifung geht bei dem Benzoperoxyd, wie schon erwähnt, sehr glatt von statten und es bieten sich in dieser Hinsicht keine Schwierigkeiten. Freilich gelingt es nach unserer Ansicht nicht, mit dieser Methode in unserem Falle so genaue Resultate zu erzielen, wie auf dem maßanalytischen Wege, ganz abgesehen daron, daß sie verhältnismäßig umständlich und zeitraubend ist. Jedoch sind die auf diesem Wege erhaltenen Resultate einwandfrei.

Zwei Versuche ergaben folgende Zahlen:

$$
\text { Analysse No. }
$$

I.

II.

Abgewogenes Benzoperoxyd, Gramm . . 0,3980 0,4251

Gefundene Benzoesäure, Gramm . . . . 0,3994

Gefundenes Benzoperoxyd, Gramm . . 0,3958

Prozent 


\section{Ueber die Verwendung des Benzoperoxyds.}

Veber die Verwendung des Benzoperoxyds in technischer, physiologischer und medizinischer Hinsicht liegen verschiedene Angaben vor.

In der Technik hat sich das Benzoperoxyd vor allem als ölbleichendes Mittel bewährt. Der Vorteil des Verfahrens liegt darin, daß sich bei der Bleichung kein Niederschlag bildet und deshalb Oelverluste vermieden werden können. Das entstandene Anhydrid soll vollkommen unschädlich sein und kann mit Wasserdampf als Benzoesäure abgetrieben werden. Um einen Effekt zu erzielen, sind nach Dr. L ü d e $\left.\mathrm{c} \mathrm{k} \mathrm{e}^{\mathrm{l}}\right)$ auffallend geringe Mengen $(0,1-0,2 \%)$ verwendbar. Nur Palmöl erfordert 0,4\%. Die Lösung des Peroxyds in Fett gelingt bei $70-80^{\circ} \mathrm{C}$.; das Fett wird dann auf $95-100^{\circ} \mathrm{C}$. erwärmt und $15-20$ Minuten, oft auch schneller, ist die Bleichung vollendet. Erdnußöl, Maisöl, Olivenöl, Senföl und Sesamöl lassen sich damit gut bleichen. Leinöl wird bei Behandlung mit Benzoperoxyd braunrot. Ein solches Leinöl besitzt einen hübschen Bronzeton; es ist zwar für die Seifenindustrie nicht verwendbar, dagegen sehr brauchbar zur Herstellung von Lacken und Farben. Rührt man mit diesem Oel eine weiße oder eine andere fette Farbe an, so tritt ein besonders weißer Ton ein und die Brillanz der Farbe nimmt zu.

Das grüne Sulfuröl schlägt, mit $0,2 \%$ Benzoperoxyd behandelt, seine Farbe in Braun um. Die daraus hergestellten Seifen sind hellbraun, heller als die aus mit Dichromat gebleichtem Sulfuröl hergestellten Seifen. Setzt man eine mit Benzoperoxyd behandelte Seife dem Licht und der Luft aus, so wird sie vollständig weiß, und ist dann nicht mehr zu unterscheiden von der besten Olivenölseife. Auch für Talg und tierische Fette empfiehlt sich diese Art von Bleichung, wobei das Präparat auch noch eine desodorierende Wirkung ausübt.

Das Superoxyd wird unter dem Namen ,Lucidol“" von den „Vereinigten Chemischen Werken" zu Charlottenburg in den Handel gebracht. Auch als Zusatz zu Toiletteseifen ist das Benzoperoxyd schon versucht worden, ebenso als haarbleichendes Mittel.

Die ersten Versuche über die physiologische Wirkung des Körpers stellte Dr. $\mathrm{Hell} \mathrm{in}^{2}$ ) aus Petersburg an. Diese Versuche ergaben, daß Dosen von $8 \mathrm{~g}$ Benzoperoxyd für Hunde und

1) Neueste Erfindungen und Erfahrungen 38 (1911), 74.

2) Pharm. Zentralh. 40 (1899), 210. 
Kaninchen ungiftig sind. Später beschäftigte sich Dr. (. F r e $y^{1}$ ) mit der keimtötenden Kraft des Stoffes. Aus nachstehender Tabelle sind die erhaltenen Resultate ersichtlich.

Benzoylsuperoxyd tötet ab:

\begin{tabular}{|c|c|c|c|c|c|c|c|c|c|}
\hline Nach einem Zeitraum von. . & $5^{\prime}$ & $10^{\prime}$ & $15^{\prime}$ & $\left|20^{\prime}\right|$ & $30^{\prime}$ & $40^{\prime}$ & $50^{\prime}$ & $60^{\prime}$ & $90^{\prime}$ \\
\hline & 一 & - & 一 & 一 & -. & - & - & - & $x$ \\
\hline Hilzbrandbazillen & _- & - & — & 一 & . & - & — & _- & - \\
\hline Diphtheriebazillen & $x$ & $x$ & ja & $\ldots$ &.-- & - & 一 & _. & $\ldots$ \\
\hline Streptokokken & - & — & - & - & - & - & .. & 一 & ja \\
\hline Bakt. col. com. . & -- & - & ... & — & $\ldots$ & -_- & ja & - & _- \\
\hline Bac. pyocyan. & $\ldots$ & $\ldots$ & ja & - & - & — & - & $\ldots$ & - \\
\hline Bac. prodigiosus & - & -.. & 一 & $\ldots$ & $\ldots$ & - & - & ja & - \\
\hline Bac. proceus mirabilis & $x$ & $x$ & $x$ & ja & $\ldots$ & - & - & - & - \\
\hline
\end{tabular}
je $=$ Abtötung.)

(Der Strich bedeutet keine Einwirkung, $\times$ Wachstumshemmung,

In der Medizin wurde es zuerst von $A$. G. L o e w e $n$ h a r $t^{2}$ ) als brauchbares Antiseptikum empfohlen. Bei Brandwunden erwies es sich als ein schmerzstillendes, wie auch prompt heilendes Mittel, ebenso bei chronischen Beingeschwüren und bei hochgradig infizierten Wunden. Gute Dienste soll es auch bei Hautkrankheiten leisten. Bci der innerlichen Darreichung konnte der Autor weder subjektive noch objektive Symptome beobachten. Das Mittel soll sich im Organismus in Benzoesäure umsetzen und Harn als Hippursäure zum Vorschein kommen. Auch als Fixicrungsmittel für die mikroskopische Technik wurde es von $S \mathrm{t} . S \mathrm{z}$ és is i $\mathbf{i}^{3}$ ) empfohlen.

1) Pharm. Zentralh. 40 (1899), 209.

2) E. M e r c k's Jahresberichte XIX. (1905), 39.

3) E. M e r c k's Jahresberichte XXVII. (1913), 155. 